\title{
Designing fouling-resistant clay-embedded polyelectrolyte multilayer membranes for wastewater effluent treatment
}

\author{
Oishi Sanyal ${ }^{1}$, Zhiguo Liu ${ }^{2}$, Jing Yu ${ }^{1}$, Brooke M. Meharg ${ }^{1}$, Joung Sook Hong ${ }^{1}$, Wei Liao ${ }^{2}$, \\ Ilsoon Lee ${ }^{1 *}$ \\ ${ }^{1}$ Department of Chemical Engineering and Materials Science \\ ${ }^{2}$ Department of Biosystems and Agricultural Engineering \\ Michigan State University, East Lansing, Michigan 48824, USA \\ *To whom correspondence should be addressed: Email - leeil@egr.msu.edu
}

\begin{abstract}
This work explores the applicability of clay-polyelectrolyte based hybrid thin films to develop fouling resistant membrane surfaces for wastewater treatment applications. Clay nanoplatelets were layered on a commercial polyethersulfone membrane in conjunction with two oppositely charged polyelectrolytes via the aqueous-based layer-by-layer (LbL) assembly technique. These hybrid nanostructured membranes showed a high degree of fouling resistance as compared to other commercial membranes and the pure polyelectrolyte multilayer (PEM) membranes, when tested against an electrocoagulation-treated high strength wastewater. With the deposition of just 2.25 quadlayers, the clay-PEM (c-PEM) membranes demonstrated good anti-fouling properties. On crosslinking the polyelectrolytes, the c-PEM hybrid membranes showed higher reduction in the chemical oxygen demand (COD) value and enhanced fouling resistance as compared to their uncrosslinked counterparts, the pure PEM membranes (both uncross linked and crosslinked) and the bare membrane. However, the high fouling resistance of the c-PEM membranes was attained at the cost of compromising the high initial flux value of the underlying membrane. Several possible optimization strategies have therefore been suggested in this paper, which can potentially increase the flux of the modified membranes. This work, for the first time, demonstrated an attempt
\end{abstract}

This article is a part of the PhD Dissertation of Oishi Sanyal (Michigan State University, 2016) (C) 2016. This manuscript version is made available under the Elsevier user license http://www.elsevier.com/open-access/userlicense/1.0/ 
to evaluate the performance of clay-polyelectrolyte nanocomposite membranes against a real wastewater effluent.

Keywords: clay nanoplatelets; layer-by-layer assembly; membranes; fouling resistance; effluent treatment

\section{Introduction}

The shortage of drinkable water has been one of the most pressing issues of the $21^{\text {st }}$ century. The worldwide depletion in fresh water resources has created the need to purify other sources of water (like sea water) which are present in huge abundance. While desalination has grown to be a big area, the recycling and reuse of wastewater has also gained considerable attention. Various membrane based processes like ultrafiltration (UF), nanofiltration (NF) and reverse osmosis (RO) have been proposed and used for treating many different types of water sources[1-3]. While membrane technology has witnessed tremendous development over the past few decades, its application in many cases is still challenged by the issue of membrane fouling. Fouling accounts for a significant flux decline over a period of time that leads to higher pressure requirements as well as higher costs involved in membrane cleaning, replacement etc. Several research efforts have been directed towards mitigating membrane fouling like introducing changes in the process[4], bulk modification[5] and surface modification[6, 7]. Surface modification techniques have garnered considerable interest among researchers because of economical merits and ease of processing. In this work, layer-by-layer (LbL) assembly was explored as the surface modification process. It is a thin film deposition technique which involves the layering of polyelectrolytes /colloidal particles/nanomaterials via secondary molecular interactions like ionic attraction, hydrogen bonding, hydrophobic-hydrophobic interaction etc.[8]. LbL provides an effective way to tune the surface by manipulation of a number of parameters in order to satisfy the application 
requirements [9]. It is of general consensus that changing the physicochemical properties of the membrane surface like increasing the hydrophilicity and surface charge help in reducing the fouling propensity of that membrane[10]. Polyelectrolyte multilayer (PEM) membranes fabricated by the LbL assembly of polyelectrolytes on commercial membrane surfaces, therefore can well satisfy the criteria for being an effective modification process related to water treatment applications.

PEM membranes have been widely applied as high flux alternatives to commercial salt rejection membranes and have shown their potential in removing many different types of ions [1115]. However, most of these studies were confined to some simple ion-rejection tests. While most of these studies have focused on the aspects of ion rejection and ion selectivity, only a couple of studies were done on real wastewater effluents $[16,17]$. Very recently we published our work on extending the application of PEM membranes to electrocoagulation-treated high-strength wastewater effluent[18]. This work showed that PEM membranes are capable of reducing the chemical oxygen demand (COD) value of the effluent to the same level as commercial RO membranes but with much higher flux values. These membranes also exhibited superior antifouling properties than commercial membranes, when tested under dead end conditions. In this work, we focus on evaluating the anti-fouling property of these membranes with a similar effluent under cross flow conditions.

In addition to the PEM membranes, the effect of introducing nanomaterials within the LbL films was also studied. In previous studies, nanomaterials like graphene[19, 20], carbon nanotubes[21, 22] and clay[23-26] have been applied to fabricate or modify membranes. Among the many different nanomaterials, clay is easily dispersible in water and therefore can be incorporated within the LbL assembly without any additional functionalization. It is cheap, easily 
available and imparts hydrophilicity to the surface. It therefore suits the requirement of a typical surface modifier which can be used for developing large scale anti-fouling membranes. The use of clay within PEM films has been studied in great details by Grunlan et al., to fabricate gas barrier films [27-29]. The introduction of clay increased the tortuosity of the gas under consideration[30, 31]. The application of the clay-PEM hybrid films to water treatment membranes, has been comparatively rare. A couple of studies reported depositing such films on UF membrane surfaces to develop ion-rejecting membranes. Jungkyu et al., used these hybrid films for salt rejection and the incorporation of clay nanoplatelets rendered the membrane more chlorine resistant[23]. Very recently, Kovacs et al. also worked on the spray assembly of clay-polyelectrolyte quadlayers on polyethersulfone membranes for fabricating desalination membranes with higher flux than commercial RO membranes, albeit with equal salt rejection[24, 25, 30]. In fact, their work encompassed the tuning of several factors to optimize the hybrid film structure as selective layers for ion rejection and provides an excellent methodology to identify the best suited system. However, the films developed by them were only tested with a certain ion and under dead end conditions which represent markedly different conditions than testing a real effluent under tangential flow conditions. So far, these clay-PEM modified membranes have not been used with any wastewater effluent and thereby not evaluated based on their anti-fouling property. However, Hang et al., developed a composite clay-PES membrane whereby they attained higher fouling resistance with the addition of clay[26]. With this background, we believed it would be interesting to co-deposit clay nanoplatelets with polyelectrolytes using $\mathrm{LbL}$ and evaluate the performance of the modified membranes against the wastewater effluent.

In this work, the effluent from an Anaerobic Digestion (AD) reactor was fed to an electrocoagulation (EC) unit. The EC step served as a pretreatment stage for the membrane 
separation process. Based on our knowledge about the nature of this effluent, as reported in our previous publication[18], it contains humic compounds which are responsible for the organic fouling. To counter the fouling propensity of commercially available membranes, a polyethersulfone membrane was chosen as the substrate for the deposition of PEM films and clayPEM nanocomposite films. While the main focus of this work was evaluating the flux and fouling behavior of the modified membranes, we have also shown some results related to the COD removal capability of these membranes. Poly allylamine hydrochloride (PAH) and poly acrylic acid (PAA) were chosen as the polyelectrolytes and montmorillonite (MMT), as a member of the smectite group of clay minerals, was used to further modify the PEM membranes.

\section{Experimental section}

\subsection{Materials}

Poly (allylamine hydrochloride) (MW 900,000) and poly (acrylic acid sodium salt) (MW 15,000, $35 \mathrm{wt} \%$ in water) were procured from Sigma Aldrich. Sodium-Montmorillonite $\left(\mathrm{MMT}^{\left.-\mathrm{Na}^{+}\right)}\right.$clay (commercial name: Cloisite-Na ${ }^{+}$) was obtained from BYK Additives Inc. (Gonzales, TX). 2-(NMorpholino) ethanesulfonic acid (MES) Buffer was also purchased from Sigma Aldrich. N-(3Dimethylaminopropyl)-N'-ethylcarbodiimide hydrochloride (EDC) was purchased from Fisher Scientific. The commercial membranes NF 270 and BW 30 were purchased from Dow Filmtec (Midland, MI). The polyethersulfone membranes (MWCO 10,000 UE-10, Tri Sep) were provided by Sterlitech (Kent, WA). All aqueous solutions were prepared using deionized (DI) water (> 18.2 M $\Omega$ ) supplied by a Barnstead Nanopure Diamond-UV purification unit equipped with a UV source and a final $0.2 \mu \mathrm{m}$ filter. Unless specified otherwise all procedures were carried out at room temperature.

\subsection{Cross-flow filtration process setup}


A CF 042 cross flow unit (Sterlitech, Kent, WA) with an effective surface area of $42 \mathrm{~cm}^{2}$ was employed as the membrane module. The cross flow module has a rectangular geometry, the flow channel being 3.62" in length, 1.8" in width and 0.09" in depth. A positive displacement pump (Hydra-cell M03, Wanner Engineering, Minneapolis, MN) was used to deliver the feed from a conical 5-gallon feed tank. A variable speed drive (Emerson, St Louis, MO) was attached to the pump which controlled its speed. A part of the feed stream was directed back to the feed tank via a bypass valve. A back-pressure regulator was used to control the transmembrane pressure across the membrane module. The retentate stream was recycled back to the feed tank and its flow rate was measured by a Site Read Panel Mount Flowmeter (Blue-White, Huntington Beach, CA). The permeate stream was also recycled back to the feed tank. The temperature of the solution inside the feed tank was maintained at room temperature by a digital chiller (Polysciences, Warrington, PA). All the components of the cross-flow setup were obtained from Sterlitech (Kent, WA) and assembled in the lab. A detailed diagram of the setup can be found in our previous publication[15], the only difference in this case being the recycling of the permeate stream back to the feed tank.

\subsection{Effluent characterization}

The COD measurements are done following the standard procedure using COD kits purchased from $\mathrm{HACH}$ (Loveland, $\mathrm{CO}$ ). All the three types of kits (ultra-low range, low range and high range) were employed based on the COD level of the sample. A HACH reactor was used to digest the samples and the values were read using a direct reading spectrophotometer.

\subsection{Experimental protocols}

\subsubsection{Electrocoagulation (EC) protocol and preparation of the feed solution for the membrane processes}

AD effluent was obtained from the Anaerobic Digestion Research and Education Center (ADREC) at Michigan State University. Appropriate dilution (16X) was conducted so as to make the final 
Total Solids (TS) level of the effluent equal to $\sim 0.22 \%$. A column reactor with working volume of $40 \mathrm{~L}$ was designed and applied to conduct EC treatment. The key specifications for EC reactor are listed in Table 1.

Table 1. Design specifications of the electrocoagulation reactor

\begin{tabular}{|c|c|}
\hline Working volume $(\mathrm{L})$ & 40 \\
\hline Diameter of anode tube $(\mathrm{cm})$ & 29.2 \\
\hline Height of anode $(\mathrm{cm})$ & 75 \\
\hline Diameter of cathodes rod $(\mathrm{cm})$ & 6.35 \\
\hline Number of cathodes & 4 \\
\hline Surface area of cathode $\left(\mathrm{cm}^{2}\right)$ & 6700 \\
\hline
\end{tabular}

A total treatment time of 240 minutes with current level of 20 A was adopted, the procedure being modified from what was used in reported in our earlier publication [32]. Treated effluent was collected and centrifuged at $230 \mathrm{~g}$ for 10 minutes, the supernatant of which was used as the feed for the membrane filtration experiments. As the total volume of the solution required to do all the experiments was significantly high, the EC reactor was operated in multiple batches. The properties of the feed solution for the membrane processes therefore varied to some degree from one batch to another. The COD value of the feed varied between $100-220 \mathrm{mg} \mathrm{L}^{-1}$. The feed was alkaline in nature ( $\mathrm{pH} 9.04)$ with a Total Suspended Solids (TSS) value of around $17.6 \pm 0.8 \mathrm{mg}$ $\mathrm{L}^{-1}$ and a Total Dissolved Solids (TDS) value of $1334.30 \pm 123.20 \mathrm{mg} \mathrm{L}^{-1}$.

\subsubsection{Preparation of clay dispersion}

MMT-Na ${ }^{+}$clay was added to DI water at a concentration of $1 \mathrm{mg} \mathrm{mL}^{-1}$.In order to properly exfoliate the clay nanoplatelets, the dispersion was stirred for about an hour and then sonicated. A $500 \mathrm{~W}$ Tip Sonicator (QSonica, Newtown, CT) with a standard 1" horn along an additional booster, was used to sonicate the clay dispersion at $70 \%$ amplitude for 30 minutes. It was then left to being 
stirred overnight. Prior to using it in the LbL assembly, the MMT-Na ${ }^{+}$dispersion was sonicated once more for 10 minutes under the same sonication conditions as mentioned above.

\subsubsection{LbL assembly protocol}

Prior to LbL deposition, the membrane substrates were soaked in DI water overnight. The permeate sides of the membranes were covered with four alternate sheets of parafilm and aluminum foil prior to the LbL process. The required portion of the membrane was then cut out and used for the filtration experiments. This way, we were able to prevent the deposition of polyelectrolytes on both sides of the membrane. The LbL was carried out using Carl Zeiss Slide Stainer which employs a robotic arm to move the sample to different solution baths. For all polyelectrolytes, the concentration was maintained at $1 \mathrm{mg} \mathrm{mL}^{-1}$. The $\mathrm{pH}$ of the $\mathrm{PAH}$ solution was adjusted to 8.5 while the $\mathrm{pH}$ of PAA was adjusted to 3.5. The MMT-Na ${ }^{+}$solution was used without any $\mathrm{pH}$ adjustment. Two types of coated membrane systems were considered for our experiments: (1) Polyelectrolyte-based membranes (PEM membrane) and (2) Polyelectrolyte-clay hybrid membranes (c-PEM membrane). For the polyelectrolyte-based membranes, the usual bilayer type of assembly was used. While, for the c-PEM membranes, the polyelectrolyte and clay layers were deposited in the form of quad layer assembly.

Bilayer assembly: $[\mathrm{PAH} / \mathrm{PAA}]_{\mathrm{m}} ; \mathrm{m}=$ number of bilayers

Quadlayer assembly: $[\mathrm{PAH} / \mathrm{PAA} / \mathrm{PAH} / \mathrm{MMT}]_{\mathrm{n}} ; \mathrm{n}=$ number of quadlayers

The dipping time in each polyelectrolyte solution was 10 minutes followed by three consecutive DI water rinsing steps ( 2 minutes, 2 minutes and 1 minute respectively). The dipping time in MMT$\mathrm{Na}^{+}$solution was 20 minutes, followed by three rinsing steps. For the c-PEM membranes, the sample was sonicated in an ultrasonic bath sonicator, following the deposition of each quad layer. For the PEM samples, the sonication was done after every bilayer. 


\subsubsection{Chemical cross-linking using EDC}

The EDC solution was prepared in a $50 \mathrm{mM}$ MES Buffer solution at a concentration of 50 $\mathrm{mg} \mathrm{mL}^{-1}$ and $\mathrm{pH}$ of 5.5. Following the deposition of the multilayers on its surface using the aforementioned LbL protocol, the modified membrane was dipped in the EDC solution using the slide stainer for 60 minutes with continuous agitation. The samples were then subject to three consecutive DI water rinsing steps, each of them being of 15 minutes duration, and then followed by 5 minutes of sonication.

\subsubsection{Cross flow filtration protocol}

All membranes to be tested were soaked in DI water overnight. They were then compacted in the cross flow system for at least 12 hours by just passing DI water across the membranes. Following the compaction step, the membranes were tested against the EC effluent for 48 hours under 5 bar transmembrane pressure and cross flow velocity of $1 \mathrm{~L} \mathrm{~min}^{-1}$. The flow rate measurements were done at time $\mathrm{t}=0,0.5,1,2,3,5,8,12,24,30,36$ and 48 hours for all membranes. For each type of membrane, at least two replicates were tested. After every experiment, the setup was cleaned thoroughly with DI water for at least three times. For each experiment, the feed samples and the permeate samples were analyzed for their COD level and the rejection was calculated based on these values.

\subsubsection{Measurement of film thickness}

The thickness of the films was measured by Dektak Surface Profiler. For measurement purposes, the films were deposited on glass slides. Prior to LbL deposition, the bare glass slides were treated with $\mathrm{O}_{2}$ plasma for 20 minutes using a Harrick plasma cleaner (Harrick Scientific Corporation, Broading Ossining, NY) at $30 \mathrm{~W}$ RF power under 100 millitorr vacuum pressure. Measurements 
were taken at minimum three different spots in each glass slide and for each type of film, at least two replicates were tested.

\section{Results and discussion}

\subsection{Performance of commercial membranes}

Three commercial membranes were tested for their performance in terms of solution flux and COD removal- a) a polyethersulfone (PES) UF membrane, b) a NF membrane and c) a RO membrane. The flux profiles of the commercial membranes are shown in Figure 1a. The PES membrane, with a MWCO of 10,000 had the highest flow rate, as would be expected. Its flux profile was that of a typical UF membrane wherein there was a drastic drop in the value during the initial few hours, followed by progressively lower flux declines for the rest of the time period. These membranes usually have slightly hydrophobic surfaces and therefore have high fouling propensities[7]. The NF 270 membrane is a polypiperazinamide-based semi-aromatic NF membrane primarily meant for removing small sugar molecules, divalent/multivalent ions etc. The BW 30 membrane is a polyamide thin-film composite RO membrane, primarily aimed at removing salts from brackish water $[15,33,34]$. The flux trend of these membranes was in accordance with their MWCOs. All three membranes, under consideration showed quite high fouling propensities. The PES membrane, being the least hydrophilic one suffered the maximum fouling, followed by NF 270 and BW 30. The fouling propensity was quantitatively estimated as the ratio of the flux at that particular time $\left(\mathrm{J}_{\mathrm{s}}\right)$ to the initial flux $\left(\mathrm{J}_{0}\right)$. This profile can be seen in Figure $1 \mathrm{~b}$.

For the PES membranes, the COD reduction varied within a range of 5-50\% because of the broad pore size distribution of such membranes. For the NF 270 and BW 30 membranes, the COD value of the permeate streams dropped below the detection limit of the COD Spectrophotometer, therefore indicating very low values. 


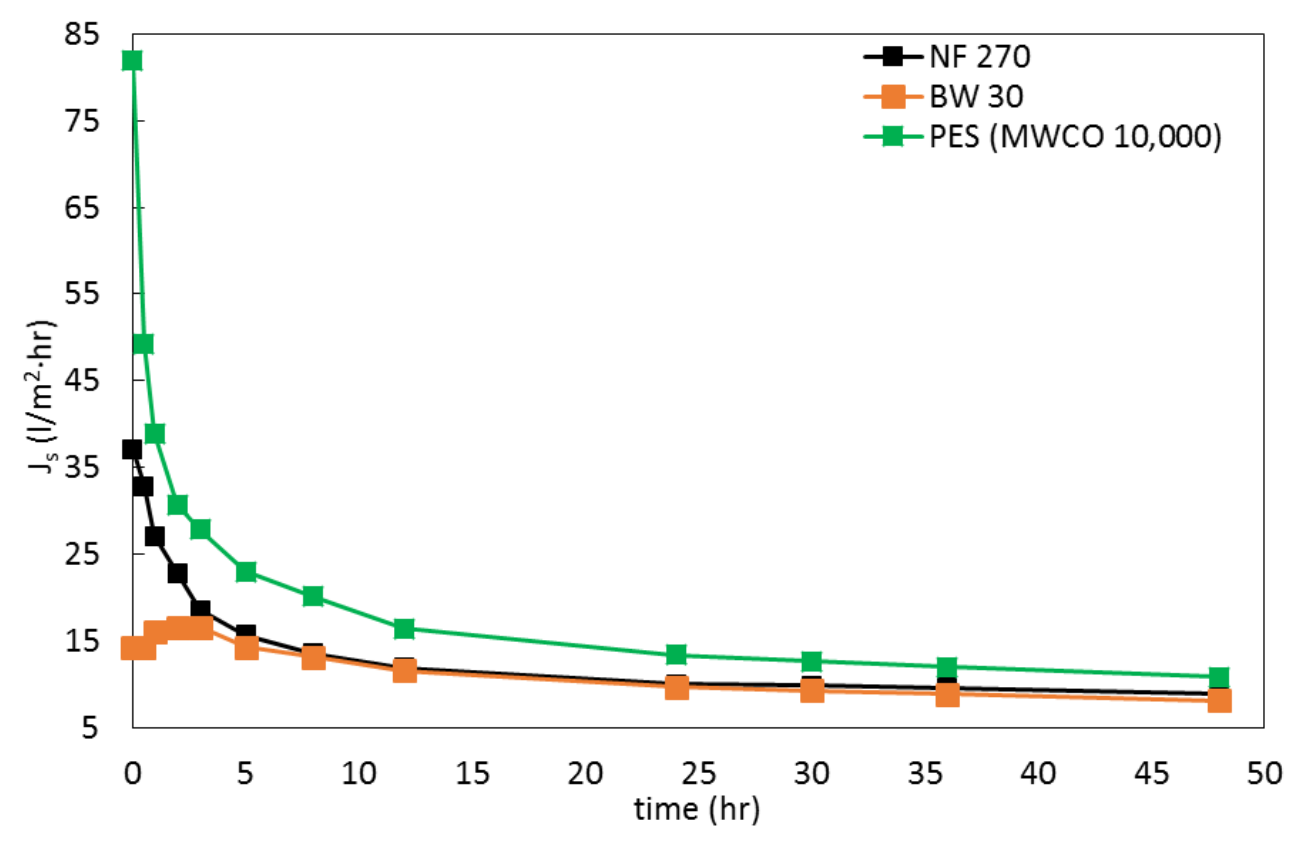

(a)

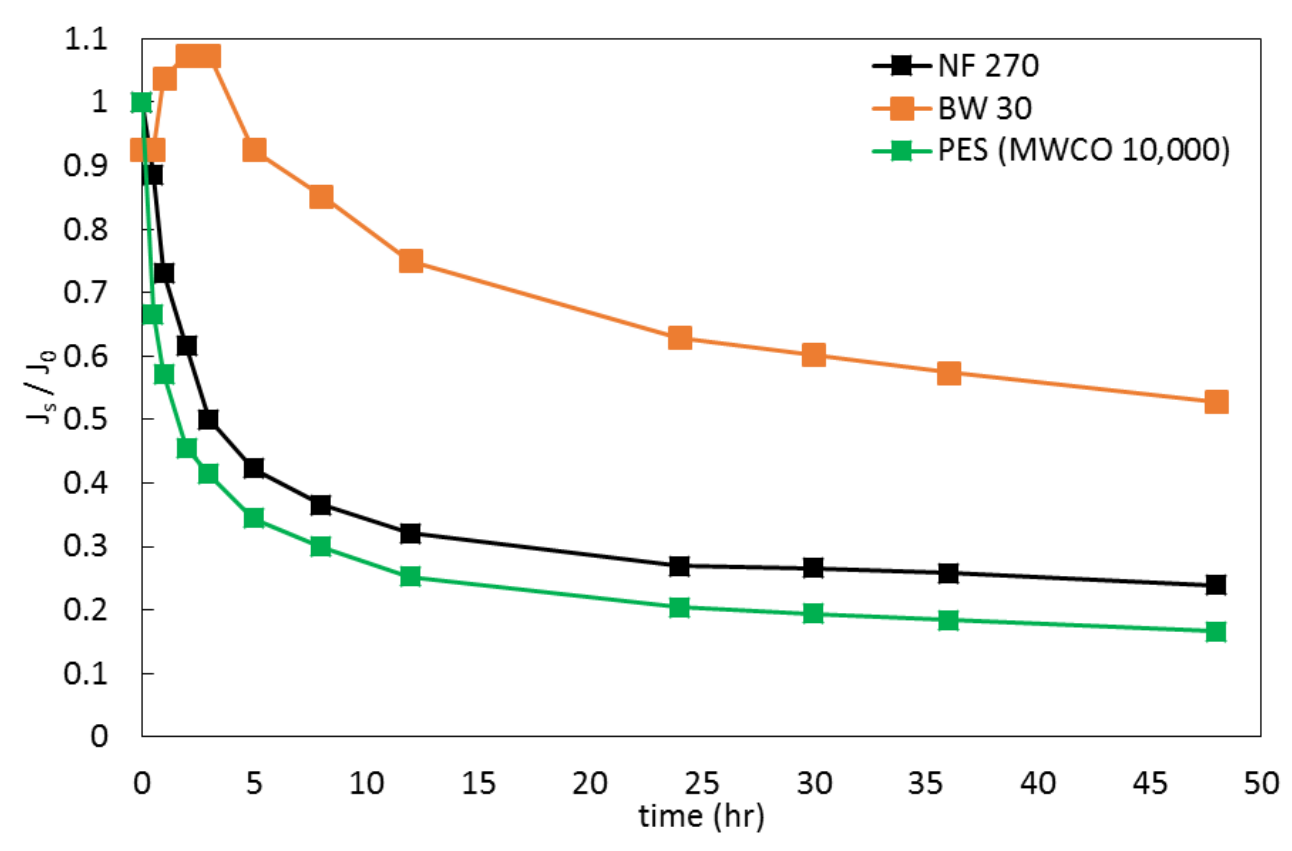

(b)

Figure 1. Performance of the three commercial membranes as a function of time in terms of (a)the solution flux and (b) the fouling propensity expressed as the ratio of solution flux at that particular time to the initial flux of the membrane. 
The PES membrane was chosen as the substrate for further modification because of its high initial flux. Modifying the surfaces of NF 270 or BW 30 would have led to membranes with extremely low fluxes. In the following sections of this paper, the performance of the PES membrane, modified with PEM films and c-PEM hybrid films have been discussed in great details.

\subsection{Performance of PEM membranes}

In our previous publication, the possibility of using PEM membranes in conjunction with electrocoagulation to reduce the COD level of a high-strength effluent was shown. These results were obtained using a dead-end filtration setup whereby these membranes also showed better antifouling properties than the commercial membranes[18]. In this study, we focused specifically on the anti-fouling property of such membranes under cross flow conditions. Based on the results from our previous publication, the PAH ( $\mathrm{pH} 8.5)$ /PAA ( $\mathrm{pH} 3.5)$ system with PAH as the outermost layer was chosen. Unlike the previous work, till this stage, the polyelectrolytes were not chemically crosslinked and we simply tested this system with different number of bilayers $(2.5,4.5,5.5$, and 6.5). Once the most optimized system was identified, the effect of crosslinking was studied on that particular system and this has been discussed in the later sections of this manuscript. With increasing number of bilayers, we assumed, there was increased surface coverage. Given that the underlying PES substrate was highly porous, a uniform coverage necessitated a certain minimum number of bilayers. The results, as evident from Figure 2, are in agreement with our hypothesis. With the deposition of just 2.5 bilayers, the flux of the modified membrane remained practically unchanged from that of the underlying bare membrane. The change was more obvious from 4.5 bilayers onwards. The flux data of these membranes modified with different number of bilayers are shown in Figure 2a. The anti-fouling property of the modified membranes improved with increasing the number of bilayers. However, even after 6.5 bilayers the membranes still showed 
significant fouling propensity as can be seen in Figure $2 \mathrm{~b}$. Besides, with the deposition of 6.5 bilayers, the flux of the modified membrane had already dropped to a very low value (10\% of that of PES membrane). Without continuing to deposit additional bilayers, we resorted to modify the PEM membranes by incorporating clay nanoplatelets within the layers.

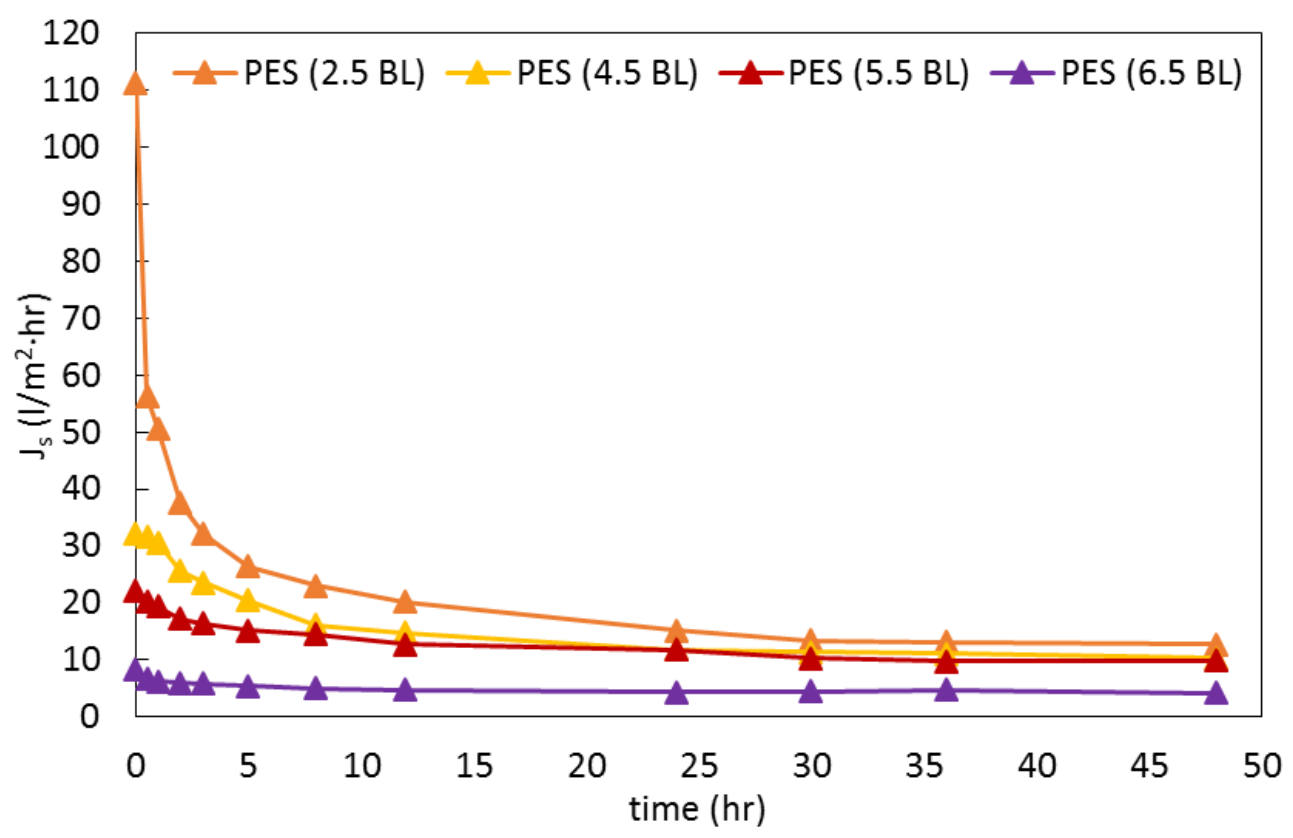

(a) 


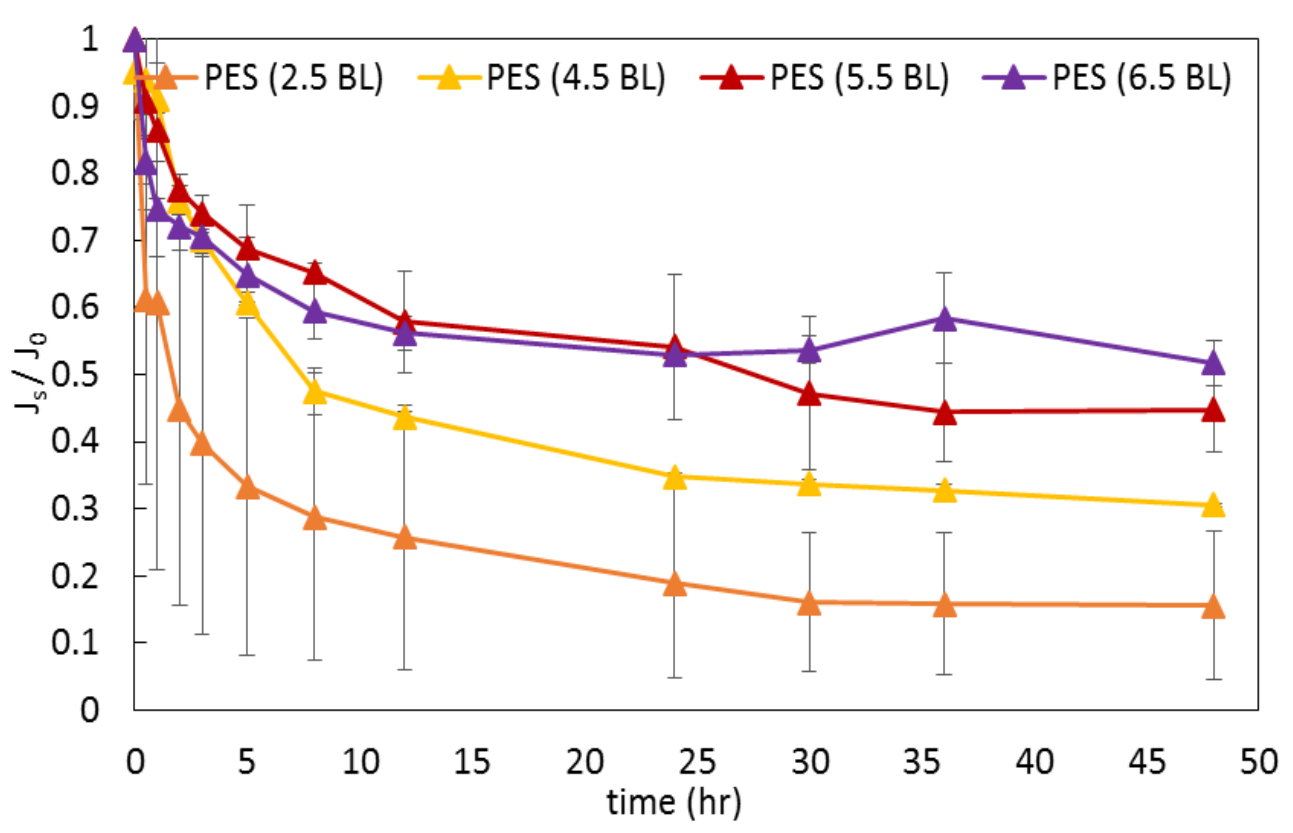

(b)

Figure 2. Performance of PEM membranes as a function of time in terms of (a) the solution flux and (b) the fouling propensity expressed as the ratio of solution flux at that particular time to the initial flux of the membrane.

PES ( $n$ BL) refers to PES membrane modified with $n$ number of bilayers where $n=2.5,4.5,5.5$ and 6.5 .

\subsection{Performance of c-PEM membranes}

The c-PEM hybrid films were formed by incorporating MMT-Na ${ }^{+}$clay nanoplatelets within the PEMs in a quadlayer fashion, as described in the experimental section. PAH and PAA were used in the same $\mathrm{pH}$ conditions as in the case of pure PEM films and they were terminated with PAH as the outermost layer. Among the types of clay (laponite, montmorillonite and vermiculite) commonly used in literature[35], montmorillonite was chosen for our experiments as we speculated that, with its intermediate platelet size, it would provide the optimum balance between high flux and high removal. In the quadlayer assembly, the consecutive clay layers are separated from each other by three alternately charged polyelectrolyte layers. According to previous research findings, the distance between the clay layers plays a very important role in the performance of 
such films[36]. Several configurations have been reported in literature like the usual bilayer configuration[25, 30, 37], tri-layer[38], quad-layer (sometimes also referred to as tetra-layer), hexa-layer and even octa-layer configurations[35]. Based on the results reported by Kovacs et al., the quadlayer assembly was chosen as it provides optimum spacing between the individual clay layers as well as optimum percentage of clay incorporation within the hybrid films[30]. As evident from the flux profile in Figure 3a, with the deposition of each quadlayer, the flux decreased considerably. However, the fouling propensity of the quadlayer-coated c-PEM membranes was significantly better than the bare membrane or even the PEM membranes. This can be seen in Figure $3 \mathrm{~b}$. With the deposition of 1.25 quadlayer (the 0.25 signifies the outermost PAH layer) the fouling propensity of the membrane showed a marked improvement. Moving from 1.25 to 2.25 quadlayers, the anti-fouling property showed the most significant improvement and with another additional quadlayer, it attained perfection. However, with each quadlayer deposition there was a significant drop in the flux and with just 3.25 quadlayers, the flux of the c-PEM membrane to 2.14 $1 / \mathrm{m}^{2} \cdot \mathrm{hr}$ from a value of $81.97 \mathrm{l} / \mathrm{m}^{2} \cdot \mathrm{hr}$ for the bare PES membrane.

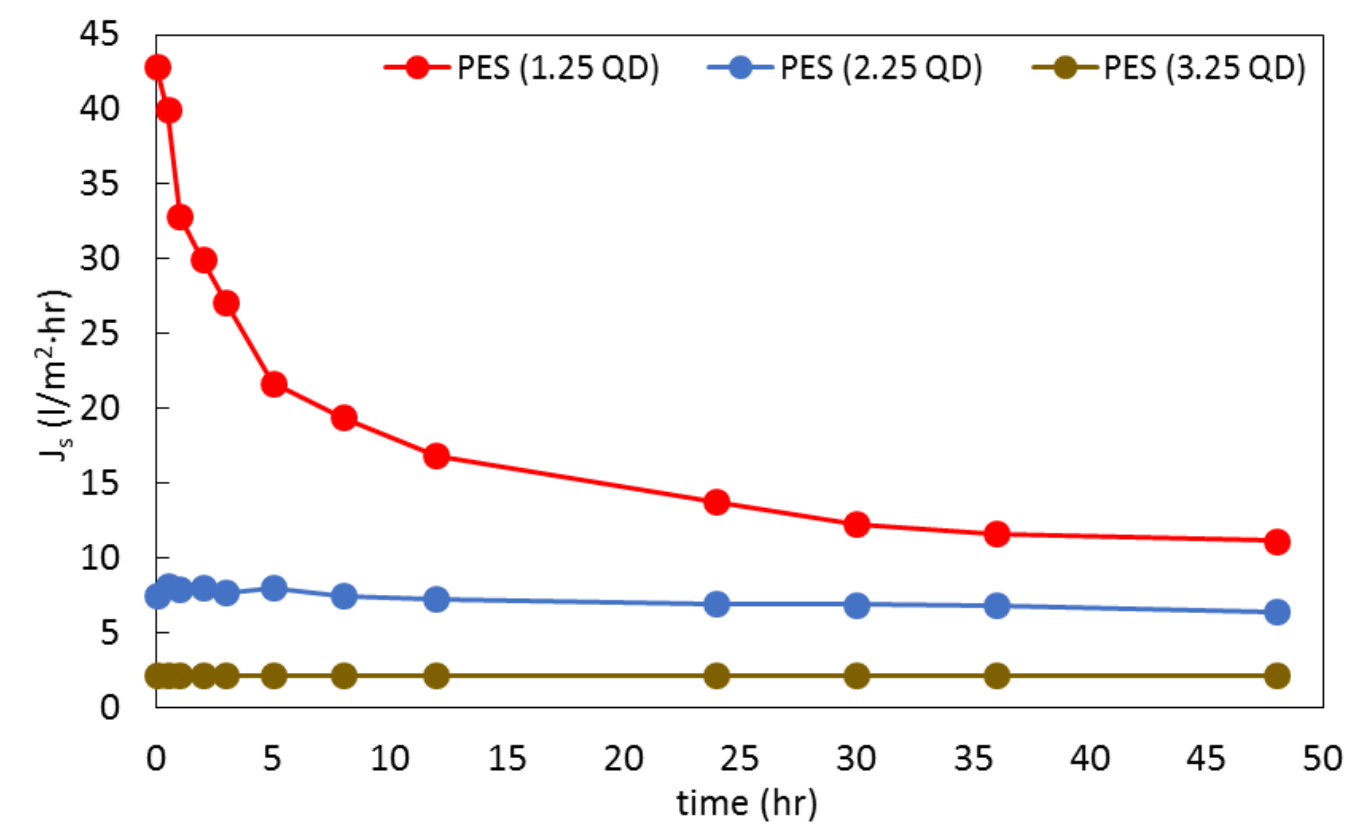


(a)

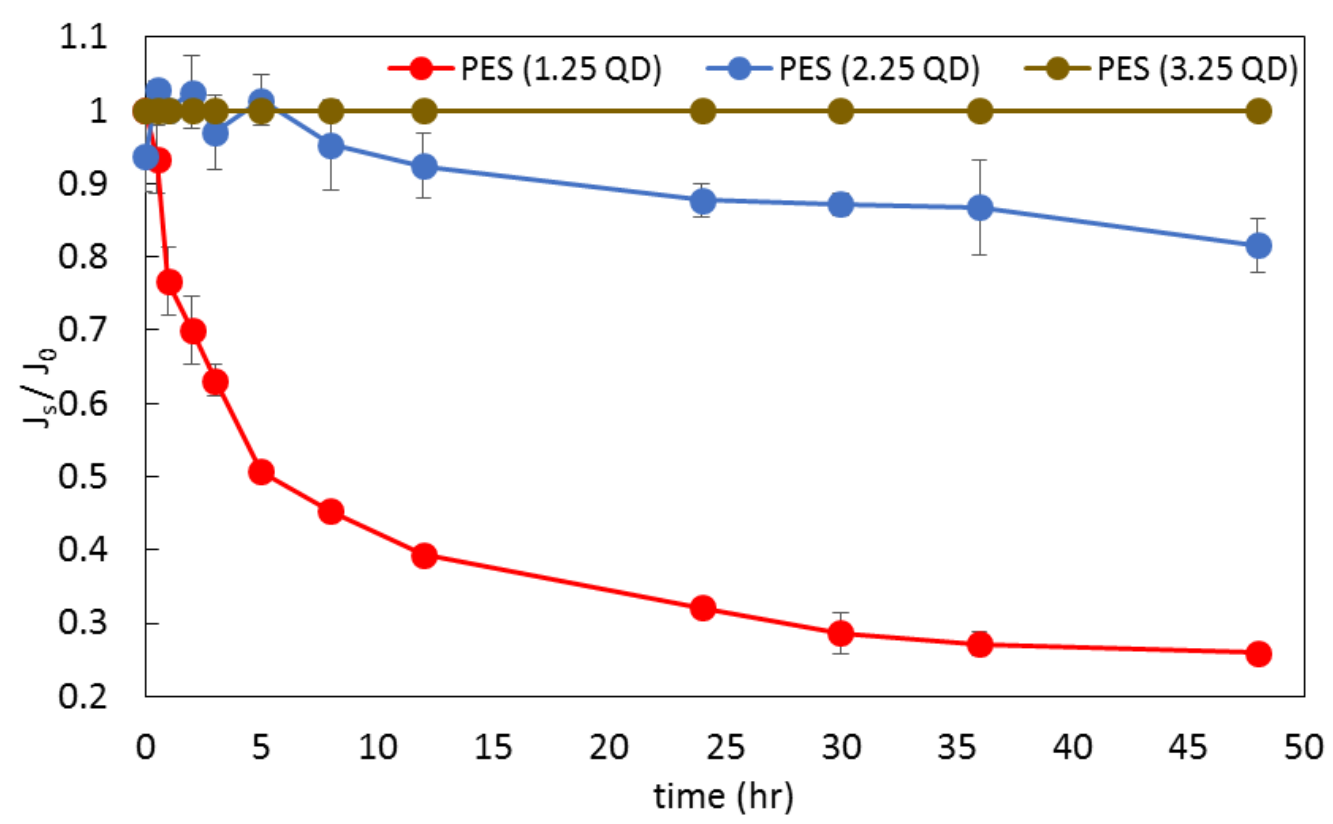

(b)

Figure 3. Performance of c-PEM membranes as a function of time in terms of (a) the solution flux and (b) the fouling propensity expressed as the ratio of solution flux at that particular time to the initial flux of the membranes.

PES (n QD) refers to PES membrane modified with n number of quadlayers where $\mathrm{n}=1.25,2.25$ and 3.25 .

\subsection{Overall picture}

In this section we compare the results from all the different membranes - the three commercial membranes, the PEM membranes and the c-PEM membranes, as can be seen in Figure 4. The modification of the bare PES membrane inevitably caused a decrease in its initial flux, irrespective of being modified by PEMs or c-PEM films, as can be seen in Figure 4a. The c-PEM hybrid membranes, when compared against the corresponding PEM membranes with the same number of layers, showed lower fouling propensity, albeit at the cost of lower initial flux. This can be seen in Figure $4 \mathrm{~b}$. For example, the 1.25 quadlayer system showed higher fouling resistance but lower initial flux than 2.5 bilayer (both have 5 layers in total) system. The same was observed for the 
2.25 quadlayer/4.5 bilayer (9 layers) and 3.25 quadlayer/6.5 bilayer (13 layers) systems. However, a closer look at Figure 4 reveals that the 6.5-bilayer (13 layers) coated PEM membrane had the same initial flux as the 2.25 quadlayer (9 layers) coated c-PEM membrane. But after 48 hours of testing, for the 6.5 bilayer system, the flux dropped to around $60 \%$ of its initial value, while the 2.25 quadlayer system retained almost $90 \%$ of its initial flux. It means that a c-PEM membrane does significantly better in retaining its flux when compared against a pure PEM membrane with the same initial flux. In other words, if we were to attain the same degree of fouling resistance as 3.25 quadlayers, we would need even more than 6.5 bilayers of PEMs (exact number not determined) and this would lead to further lowering of the initial flux value beyond what is currently offered by the 3.25 quadlayer system. The 2.25 quadlayer system also had very similar initial flux value as the commercial RO membrane (BW 30) but its anti-fouling property is much better than the latter. Inclusion of clay nanoplatelets within the PEMs therefore proved to be advantageous in terms of flux and number of layers required. The 3.25 quadlayer system showed the best performance in terms of fouling resistance but it retained only $2.6 \%$ of the initial flux of the bare membrane which therefore doesn't make it suitable for practical purposes.

The exact mechanism by which the addition of clay nanoplatelets within the PEM films served to reduce the fouling tendency of the membranes is not very well understood. While arguments could be put in favor of enhanced surface charge, hydrophilicity etc., some preliminary contact angle tests did not reveal any particularly discernible change. A detailed mechanistic study was beyond the scope of this current publication, but will definitely be looked into in our future studies. 


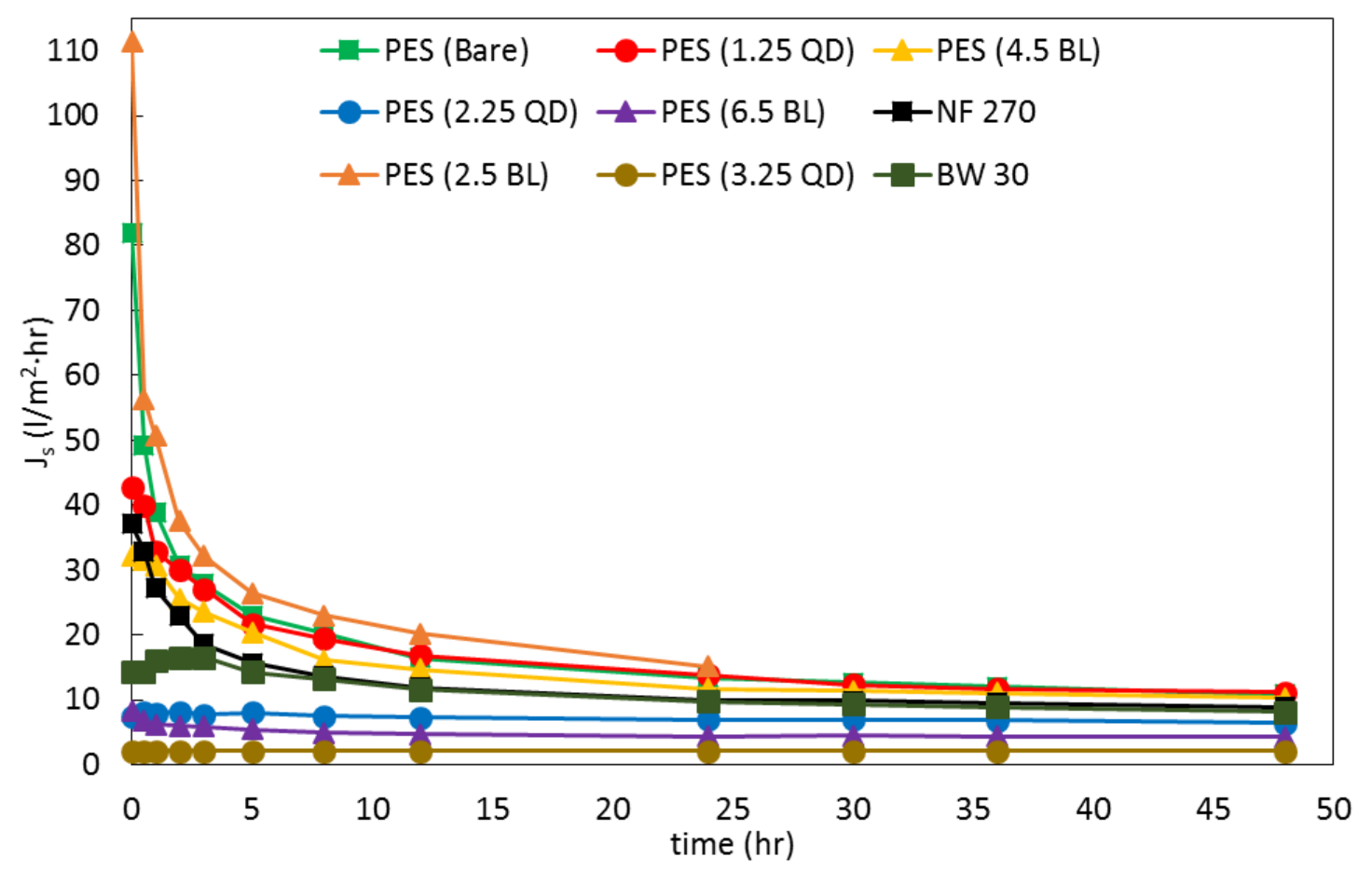

(a)

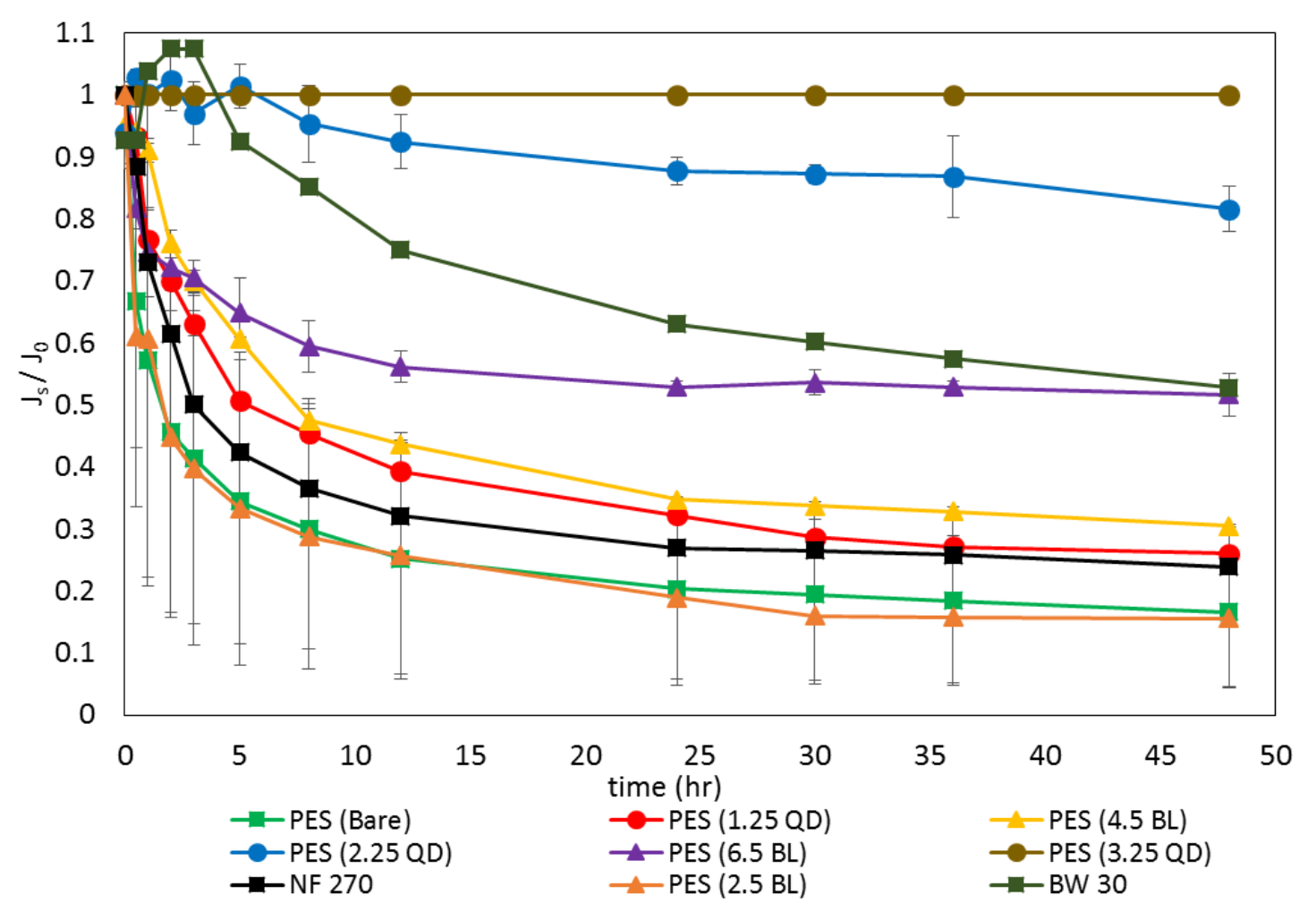

(b) 
Figure 4. Comparison in the performance of commercial membranes, PEM membranes and cPEM membranes as a function of time in terms of (a)the solution flux and (b) the fouling propensity expressed as the ratio of solution flux at that particular time to the initial flux of the membranes.

The membranes referred to as PES ( $\mathrm{n}$ BL) $(\mathrm{n}=2.5,4.5$ and 6.5) are the PEM membranes and ones referred to as PES ( $\mathrm{n} Q \mathrm{QD})(\mathrm{n}=1.25,2.25$ and 3.25) are the c-PEM membranes.

The thickness data of the PEM film as well as the c-PEM hybrid films are shown in Table 2. The trend of increase in thickness with the increase in bilayers/quadlayers deposited on the surface is quite expected and the flux profile can also be related quite well with the thickness date. Although the 6.5 bilayer system had higher thickness than the 2.25 and 3.25 quadlayer systems, as shown before, its flux was equal to that of the 2.25 quadlayer system and higher than the 3.25 quadlayer system. We believe, this might be due to suppression of the polymer interdiffusion by the clay nanoplatelets that leads to lower thickness values of the c-PEM films. Then again, these nanoplatelets introduced a high degree of tortuosity of pathway which explains the low solution flux across the c-PEM membranes. 
Table 2. The thickness values of the PEM films and c-PEM films

\begin{tabular}{ccc}
\hline Modified film type & Film assembly & Film thickness (nm) \\
\hline & PES (2.5 BL) & $46.67 \pm 6.50$ \\
PEM films & PES (4.5 BL) & $86.00 \pm 14.14$ \\
& PES (6.5 BL) & $182.67 \pm 2.52$ \\
& & \\
& PES (1.25 QD) & $73.37 \pm 6.18$ \\
c-PEM films & PES (2.25 QD) & $108.47 \pm 7.43$ \\
& PES (3.25 QD) & $131.50 \pm 4.95$ \\
\hline
\end{tabular}

\subsection{Effect of EDC cross-linking on the performance of membranes}

The PEM membranes and the c-PEM membranes showed a considerable reduction in the flux due to blocking of the pores of the underlying membrane. The COD reduction was however, not significantly improved as compared to the bare membrane (data not explicitly shown). This necessitated the polyelectrolytes to be crosslinked in order to limit the passage of some very fine, dissolved contaminants. Chemical crosslinking with EDC was preferred over thermal treatment due to the very apparent deformation of the underling substrate under high temperature.

\subsubsection{Effect on flux and anti-fouling property}

The effect of crosslinking was studied for PEM membranes as well as c-PEM membranes. For the PEM membranes, the 5.5 bilayer system was chosen as it had almost similar anti-fouling property as the 6.5 bilayer system with a slightly higher initial flux. For the c-PEM membranes, the system comprising of 2.25 quadlayers was chosen as the most optimum system as its flux was much higher than the 3.25 quadlayers system and its fouling resistance was very good. As expected, there was 
a drop in the flux in both cases as can be seen from Figure 5. The drop in flux was more significant in case of the PEM membranes ( 50\%) compared to the c-PEM membranes $(\sim 37 \%)$. This can be explained by the fact that the presence of clay nanoplatelets limits the interdiffusion of the polyelectrolytes to some extent and therefore limits the amine groups of PAH and acid groups of PAA from reacting with each other to the same extent, as in the case of just PEM membranes. It was also interesting to note that for both the systems the flux decline was reduced due to crosslinking, a phenomenon which has not been studied till date. In fact, the anti-fouling property of the crosslinked 2.25 quadlayer membrane was as good as that of the uncrosslinked 3.25 quadlayer system, but with higher initial flux. This, therefore opens up a new optimization strategy to improve the anti-fouling property of polyelectrolyte-based membranes.

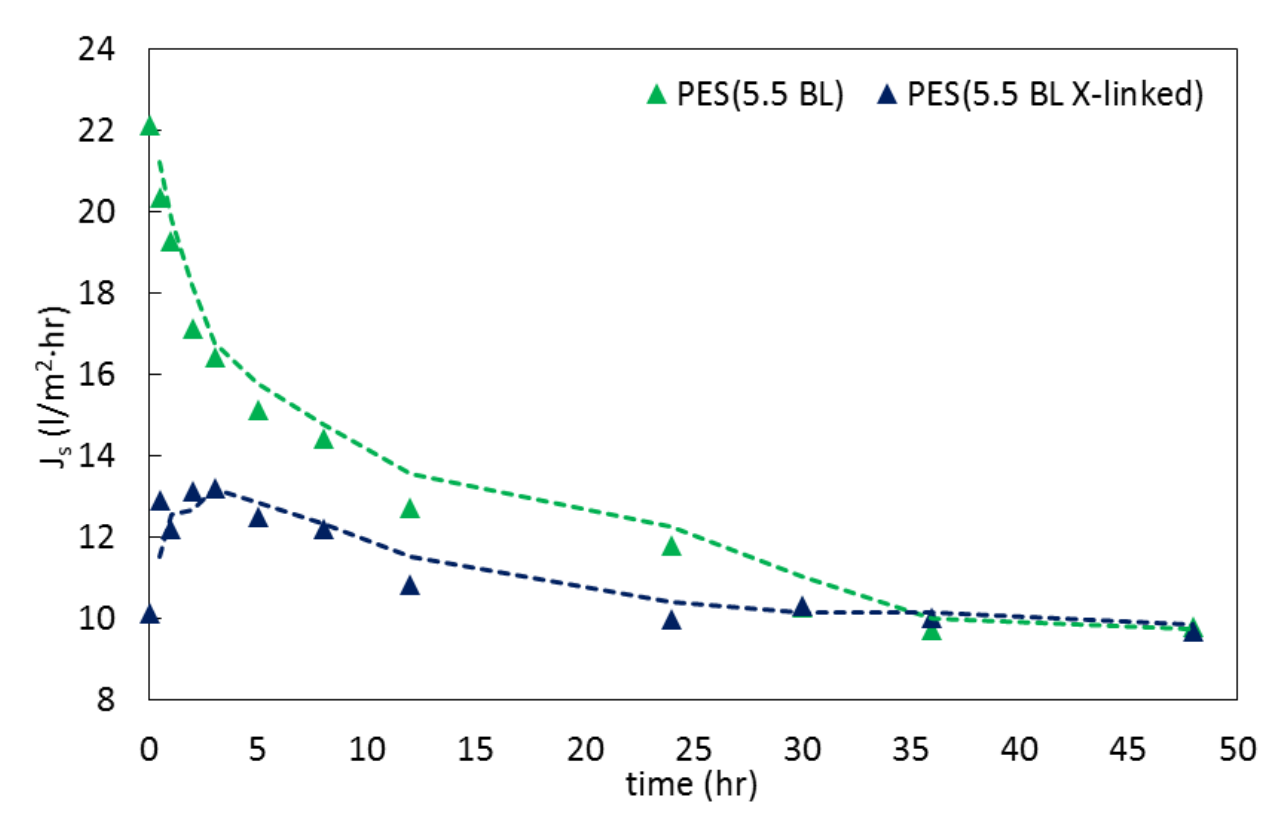

(a) 


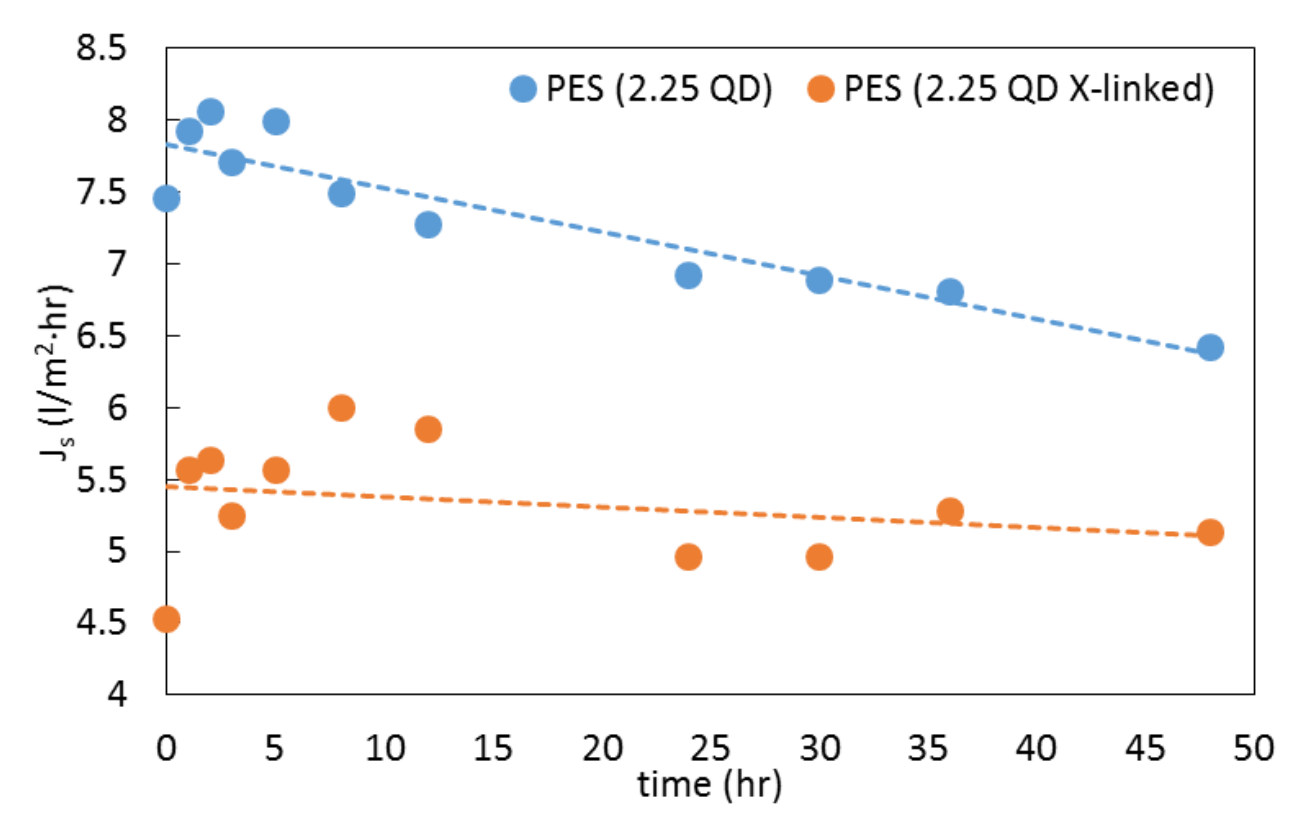

(b)

Figure 5. Effect of cross-linking on the solution flux of a) 5.5 bilayer PEM membrane and b) 2.25 quadlayer c-PEM membranes.

\subsubsection{Effect on COD reduction}

The main rationale behind crosslinking the multilayers was to improve the COD reduction of the modified membrane. However, on crosslinking the 5.5-bilayer modified membrane, there was no significant change in rejection compared to its uncrosslinked counterpart. On the other hand, the PES membrane modified with 2.25 quadlayers of PEM-clay hybrid films showed a significant improvement, as is evident in Figure 6. This result therefore provides another justification to the use of c-PEM membranes over PEM membranes. The clay nanoplatelets provided an additional barrier to the passage of unwanted contaminants, as has been observed in the case of ion rejection and gas barrier films. However, the reduction showed a declining trend, dropping to very low values after 24 hours, the cause(s) behind which are not exactly known to us. One possible reason could be the phenomenon of concentration polarization, which can be minimized by increasing the 
crossflow rate and other hydrodynamic conditions[39]. These experiments however gave us a fair indication that it is possible to get high COD removal by crosslinking the 2.25 quadlayer system.

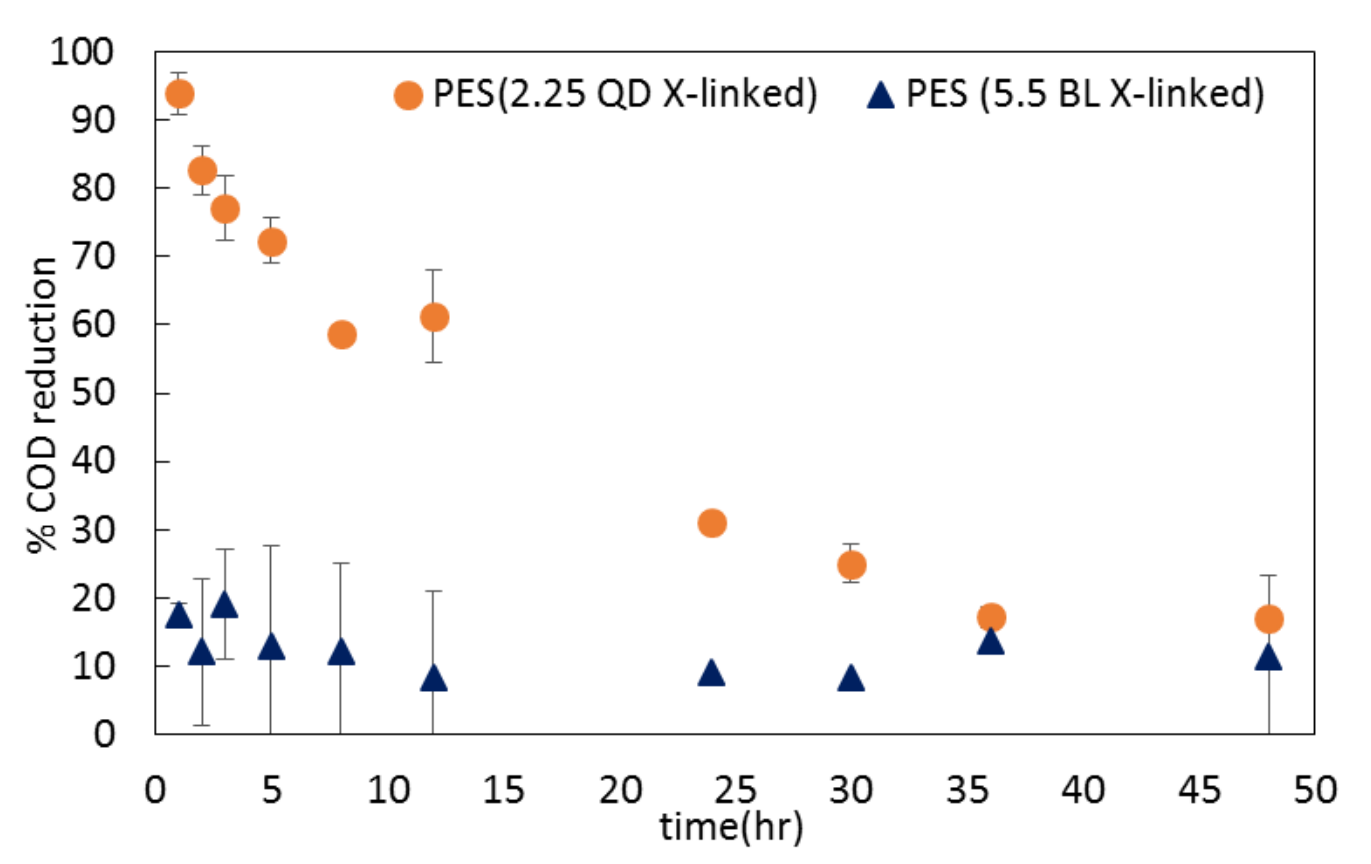

Figure 6. Comparison in the COD reduction of the cross-linked 5.5 bilayer PEM membrane and crosslinked 2.25 quadlayer c-PEM membrane.

In summary, the addition of clay nanoplatelets within PEM films proved to be advantageous in a number of ways. First, lesser number of layers were required to modify the surface which implied higher flux and lower processing times for film fabrication. Secondly, although the primary focus of this work was on developing anti-fouling surfaces, it was found that a simple cross-linking step enabled these films to reduce the COD level of the effluent to very low values. In fact, due to the widely reported barrier property of clay nanoplatelets, the c-PEM films were much more efficient than the PEM films in terms of COD reduction. More work remains to be done in the area of increasing the initial flux of these membranes. 


\section{Conclusion and recommendations for future work}

For the first time, nanostructured c-PEM hybrid films were used to modify membranes for treating a real wastewater solution, as a part of our continued efforts to integrate modified membranes with the EC process to treat high-strength effluents. In comparison to PEM membranes, these membranes required lesser number of layers to acquire high fouling resistance and thereby was advantageous in terms of the initial flux. The membrane modified with 2.25 quadlayers was the most optimum system among the three c-PEM systems that were tried out. By crosslinking this system, the fouling resistance was further enhanced with a slight decrease in the initial flux. The COD reduction was also higher than that of the underlying membrane and the crosslinked PEM membranes. The initial flux was severely compromised because of the deposition of the c-PEM quadlayers. However, this was simply a proof-of-concept study and several manipulations to this type of assembly are possible. Previously the Grunlan research group and Hammond research group have performed some very detailed analysis to identify the factors which affect the performance of the hybrid membranes. However, since none of these films have been used for designing anti-fouling membranes and tested under tangential flow conditions, these results cannot be directly applicable to such situations. A major factor which influences the filtration performance is the amount of clay incorporated, which can be varied by tweaking some process parameters. Based on our understanding from previous literature, we have listed down some of those parameters which can help tune the amount of clay incorporated.

1. pH conditions of the polyelectrolytes: The weak polyelectrolytes like PAH and PAA have $\mathrm{pH}$-dependent ionization properties. Therefore the amount of clay incorporated within them can be varied, because at different $\mathrm{pH}$ conditions[24], the charge density of the polyelectrolytes and the number of functional groups available are very different. 
2. Clay-PEM assembly configurations: In our experiments, we followed the quadlayer assembly based on previous literature. However, some other configurations like bilayer assembly, trilayer assembly or hexa/octa-layer assembly might prove to be more beneficial for anti-fouling applications[35, 36]. By changing the clay-PEM configuration, the spacing between consecutive clay layers as well as the amount of clay within the composite film can be tuned.

3. Type of polyelectrolytes: There are a number of commercially available polyelectrolytes which can be used for LbL and therefore adds to the parameters that one can look into in order to improve the results. Among the weak polyelectrolytes, branched polyethylenimine (BPEI) /PAA also represent a pH-tunable system like $\mathrm{PAH} / \mathrm{PAA}$ and has in fact shown high oxygen barrier properties[35]. It therefore seems to be the next obvious choice of polyelectrolyte combination for future experiments.

4. Type of clay: All our experiments were done with MMT clay. However, other types of clay like laponite (LAP) and vermiculite (VMT) can be tried out, as has been done by other researchers for gas-barrier[35] and ion rejection[30]. Different clay types have varied properties like platelet dimensions, inter-platelet distance, zeta potential etc. and it is therefore needless to mention that the amount of clay incorporated within the PEM films will also be significantly different.

Apart from the above ideas, the LbL process can be shortened by reducing the dipping time involved in the buildup of each individual layer. This has been explored by the Grunlan research group[27] as well as our group[40]. While this strategy might be effective in fabricating thinner films with high fluxes, it will also make the resulting membranes more commercially lucrative. Being able to raise the permeability of these c-PEM films would open up the possibility of creating a new generation of surface-modified membranes which have all the desired properties - high permeability, high rejection as well as high anti-fouling property. 


\section{Acknowledgement}

The authors would like to thank the W.M Keck Microfabrication Facility at Michigan State University for allowing the usage of the Dektak Surface Profiler. We would also like to acknowledge the DOD Strategic Environmental Research and Development Program (DOD SERDP W912HQ-12-C-0020) for funding the entire research work.

\section{References}

[1] M. Ben-Sasson, Y. Zidon, R. Calvo, A. Adin, Enhanced removal of natural organic matter by hybrid process of electrocoagulation and dead-end microfiltration, Chemical Engineering Journal, 232 (2013) 338-345.

[2] C. Bellona, J.E. Drewes, P. Xu, G. Amy, Factors affecting the rejection of organic solutes during NF/RO treatment - a literature review, Water Research, 38 (2004) 2795-2809.

[3] W. Den, C.-J. Wang, Removal of silica from brackish water by electrocoagulation pretreatment to prevent fouling of reverse osmosis membranes, Separation and Purification Technology, 59 (2008) 318-325.

[4] H. Gu, A.R. Bartman, M. Uchymiak, P.D. Christofides, Y. Cohen, Self-adaptive feed flow reversal operation of reverse osmosis desalination, Desalination, 308 (2013) 63-72.

[5] N. Maximous, G. Nakhla, K. Wong, W. Wan, Optimization of A12O3/PES membranes for wastewater filtration, Separation and Purification Technology, 73 (2010) 294-301.

[6] C. Zhao, J. Xue, F. Ran, S. Sun, Modification of polyethersulfone membranes - A review of methods, Progress in Materials Science, 58 (2013) 76-150.

[7] A.L. Ahmad, A.A. Abdulkarim, B.S. Ooi, S. Ismail, Recent development in additives modifications of polyethersulfone membrane for flux enhancement, Chemical Engineering Journal, 223 (2013) 246-267.

[8] I. Lee, Molecular Self-Assembly: Smart Design of Surface and Interface via Secondary Molecular Interactions, Langmuir, 29 (2013) 2476-2489.

[9] O. Sanyal, I. Lee, Recent progress in the application of Layer-by-layer assembly to the preparation of nanostructured ion-rejecting water purification membranes, Journal of Nanoscience and Nanotechnology, 14 (2014) 2178-2189.

[10] L. Zou, I. Vidalis, D. Steele, A. Michelmore, S. Low, J. Verberk, Surface hydrophilic modification of RO membranes by plasma polymerization for low organic fouling, Journal of Membrane Science, 369 (2011) 420-428.

[11] S.U. Hong, O.Y. Lu, M.L. Bruening, Recovery of phosphate using multilayer polyelectrolyte nanofiltration membranes, Journal of membrane science, 327 (2009) 2-5.

[12] S.U. Hong, R. Malaisamy, M.L. Bruening, Optimization of flux and selectivity in Cl-/SO42separations with multilayer polyelectrolyte membranes, Journal of membrane science, 283 (2006) 366-372.

[13] S.U. Hong, R. Malaisamy, M.L. Bruening, Separation of fluoride from other monovalent anions using multilayer polyelectrolyte nanofiltration membranes, Langmuir, 23 (2007) 17161722. 
[14] L. Ouyang, R. Malaisamy, M.L. Bruening, Multilayer polyelectrolyte films as nanofiltration membranes for separating monovalent and divalent cations, Journal of membrane science, 310 (2008) 76-84.

[15] O. Sanyal, A.N. Sommerfeld, I. Lee, Design of ultrathin nanostructured polyelectrolyte-based membranes with high perchlorate rejection and high permeability, Separation and Purification Technology, 145 (2015) 113-119.

[16] U. Aravind, B. George, M. Baburaj, S. Thomas, A. Thomas, C. Aravindakumar, Treatment of industrial effluents using polyelectrolyte membranes, Desalination, 252 (2010) 27-32.

[17] M. Baburaj, C. Aravindakumar, S. Sreedhanya, A. Thomas, U.K. Aravind, Treatment of model textile effluents with PAA/CHI and PAA/PEI composite membranes, Desalination, 288 (2012) 72-79.

[18] O. Sanyal, Z. Liu, B.M. Meharg, W. Liao, I. Lee, Development of polyelectrolyte multilayer membranes to reduce the COD level of electrocoagulation treated high-strength wastewater, Journal of Membrane Science, 496 (2015) 259-266.

[19] E.N. Wang, R. Karnik, Water desalination: Graphene cleans up water, Nature nanotechnology, 7 (2012) 552-554.

[20] W. Choi, J. Choi, J. Bang, J.-H. Lee, Layer-by-layer assembly of graphene oxide nanosheets on polyamide membranes for durable reverse-osmosis applications, ACS applied materials \& interfaces, 5 (2013) 12510-12519.

[21] G.S. Ajmani, D. Goodwin, K. Marsh, D.H. Fairbrother, K.J. Schwab, J.G. Jacangelo, H. Huang, Modification of low pressure membranes with carbon nanotube layers for fouling control, Water Research, 46 (2012) 5645-5654.

[22] L. Bai, H. Liang, J. Crittenden, F. Qu, A. Ding, J. Ma, X. Du, S. Guo, G. Li, Surface modification of UF membranes with functionalized MWCNTs to control membrane fouling by NOM fractions, Journal of membrane science, 492 (2015) 400-411.

[23] J. Choi, H. Sung, Y. Ko, S. Lee, W. Choi, J. Bang, J. Cho, Layer-by-layer assembly of inorganic nanosheets and polyelectrolytes for reverse osmosis composite membranes, Journal of Chemical Engineering of Japan, 47 (2014) 180-186.

[24] J.R. Kovacs, C. Liu, P.T. Hammond, Spray Layer-by-Layer Assembled Clay Composite Thin Films as Selective Layers in Reverse Osmosis Membranes, ACS applied materials \& interfaces, 7 (2015) 13375-13383.

[25] J.R. Kovacs, P.T. Hammond, "Porous Film" US Patent Application 13/926,527 in, 2013.

[26] H. Dong, L. Wu, L. Zhang, H. Chen, C. Gao, Clay nanosheets as charged filler materials for high-performance and fouling-resistant thin film nanocomposite membranes, Journal of membrane science, 494 (2015) 92-103.

[27] Y.-H. Yang, F.A. Malek, J.C. Grunlan, Influence of deposition time on layer-by-layer growth of clay-based thin films, Industrial \& Engineering Chemistry Research, 49 (2010) 8501-8509.

[28] M.A. Priolo, D. Gamboa, K.M. Holder, J.C. Grunlan, Super gas barrier of transparent polymer- clay multilayer ultrathin films, Nano letters, 10 (2010) 4970-4974.

[29] M.A. Priolo, D. Gamboa, J.C. Grunlan, Transparent clay- polymer nano brick wall assemblies with tailorable oxygen barrier, ACS applied materials \& interfaces, 2 (2009) 312-320.

[30] J.R. Kovacs, "Engineering nanostructured selective layers for reverse osmosis membranes" $\mathrm{PhD}$ Thesis in, Massachusetts Institute of Technology, 2015.

[31] M.A. Priolo, K.M. Holder, T. Guin, J.C. Grunlan, Recent Advances in Gas Barrier Thin Films via Layer-by-Layer Assembly of Polymers and Platelets, Macromolecular rapid communications, 36 (2015) 866-879. 
[32] Z. Liu, D. Stromberg, X. Liu, W. Liao, Y. Liu, A new multiple-stage electrocoagulation process on anaerobic digestion effluent to simultaneously reclaim water and clean up biogas, Journal of hazardous materials, 285 (2015) 483-490.

[33] M. Mulder, Basic principles of membrane technology, Springer Science \& Business Media, 1996.

[34] R. Baker, Membrane Technology and Applications (3rd Edition), John Wiley \& Sons, Somerset, NJ, USA, 2012.

[35] F. Xiang, P. Tzeng, J.S. Sawyer, O. Regev, J.C. Grunlan, Improving the Gas Barrier Property of Clay-Polymer Multilayer Thin Films Using Shorter Deposition Times, ACS applied materials \& interfaces, 6 (2013) 6040-6048.

[36] M.A. Priolo, K.M. Holder, S.M. Greenlee, B.E. Stevens, J.C. Grunlan, Precisely Tuning the Clay Spacing in Nanobrick Wall Gas Barrier Thin Films, Chemistry of Materials, 25 (2013) 16491655.

[37] D.A. Hagen, L. Saucier, J.C. Grunlan, Controlling Effective Aspect Ratio and Packing of Clay with pH for Improved Gas Barrier in Nanobrick Wall Thin Films, ACS applied materials \& interfaces, 6 (2014) 22914-22919.

[38] D. Hagen, C. Box, S. Greenlee, F. Xiang, O. Regev, J. Grunlan, High gas barrier imparted by similarly charged multilayers in nanobrick wall thin films, RSC Advances, 4 (2014) 18354-18359. [39] W. Shan, P. Bacchin, P. Aimar, M.L. Bruening, V.V. Tarabara, Polyelectrolyte multilayer films as backflushable nanofiltration membranes with tunable hydrophilicity and surface charge, Journal of Membrane Science, 349 (2010) 268-278.

[40] J. Yu, O. Sanyal, A.P. Izbicki, I. Lee, Development of Layered Multiscale Porous Thin Films by Tuning Deposition Time and Molecular Weight of Polyelectrolytes, Macromolecular rapid communications, 36 (2015) 1669-1674. 